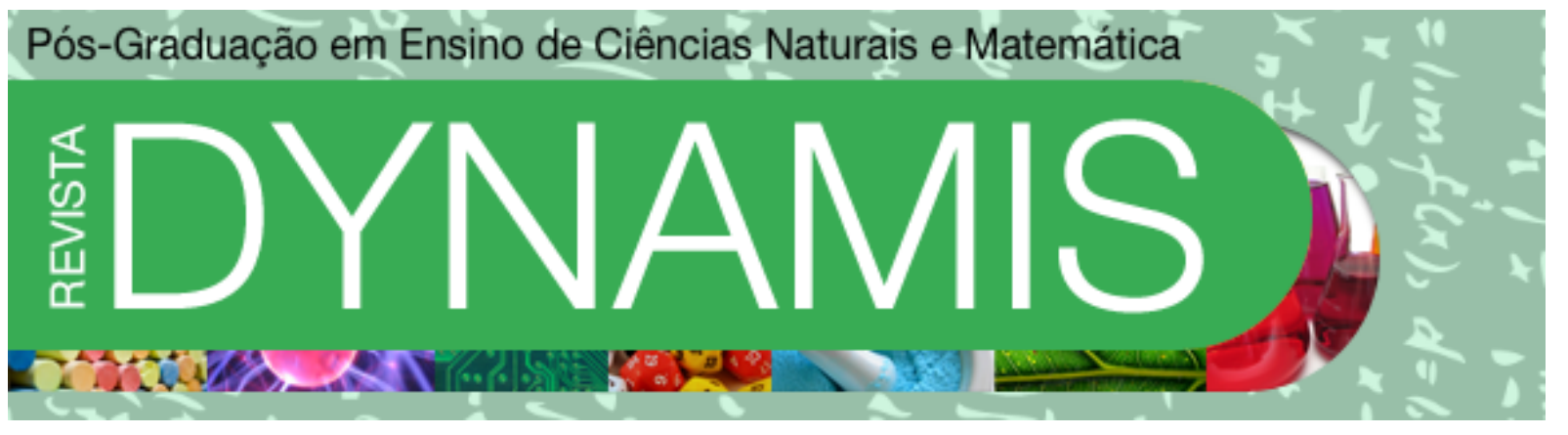

\title{
PRIMEIROS PASSOS NA ÁLGEBRA: CONCEITOS ELEMENTARES E ATIVIDADES PEDAGÓGICAS
}

First steps in algebra: Basic concepts and pedagogical activities

Janaína Poffo Possamai

Programa de Pós-Graduação em Ensino de Ciências Naturais e Matemática - PPGECIM/FURB janapoffo@gmail.com

\section{Tania Baier}

Programa de Pós-Graduação em Ensino de Ciências Naturais e Matemática - PPGECIM/FURB baier@furb.br 


\section{Resumo}

Visando refletir sobre o ensino de álgebra nos anos finais do ensino fundamental, neste trabalho, inicialmente são discutidas as diversas concepções de álgebra que se relacionam com diferentes entendimentos de variável. São focadas as dificuldades encontradas pelos estudantes no entendimento do conceito de variável e na tradução de situações escritas na linguagem usual para a linguagem algébrica. A coleta de dados foi realizada por meio de questões, envolvendo conteúdos matemáticos, organizadas seguindo como fio condutor as pesquisas de Lochhead e Mestre (1995). As atividades foram organizadas seguindo as etapas da construção histórica da álgebra, do retórico à simbolização. Neste texto são descritas as atividades realizadas e apresentadas as análises qualitativa e estatística. São tecidas considerações finais, com sugestões e recomendações destinadas aos professores que visam contribuir para a superação das dificuldades encontradas por estudantes no entendimento dos conceitos fundamentais da álgebra da educação básica.

Palavras-chave: Álgebra; ensino; Metodologia.

\section{Abstract}

In order to reflect about the teaching of algebra in the final years of primary education, this paper initially discusses the various concepts of algebra that relate to different understandings of variable. The focus is on the difficulties encountered by students in understanding the concept of variable and written translation of situations in everyday language to algebraic language. Data collection was conducted through issues involving mathematical content, organized according to the research of Lochhead and Mestre (1995). The activities were organized following the steps of the historical construction of algebra, from rhetoric to symbolization. This text describes the activities performed and it presents qualitative and statistical analysis. Some final considerations are made, with suggestions and recommendations to teachers who aim to contribute to the overcoming of difficulties encountered by students in understanding the fundamental concepts of algebra of basic education.

Keywods: Algebra; Teaching; Methodology. 


\section{Introdução}

Diferentes áreas da matemática foram privilegiadas para compor o currículo da educação básica brasileira, sendo que a álgebra tem ocupado um lugar de destaque no mundo da educação. Além das ciências exatas, em diversas áreas do conhecimento as pesquisas seguem os métodos e os conceitos da álgebra, tais como administração, economia e biologia, entre outras. Em um mundo que sofre constantes modificações, o entendimento do conceito de variável é importante para a busca da solução de situações que demandam escolhas. Assim, tanto no cotidiano das massas populares como nas diversas áreas da ciência, a compreensão do conceito de variável é fundamental.

House (1995, p. 4) constata que, para algumas áreas da ciência, o que mais interessa não é a análise e o cálculo e esclarece que o surgimento da tecnologia das calculadoras e dos computadores está exercendo forte influência sobre o crescimento de áreas como a administração, as ciências sociais e biológicas, tornando-as "altamente dependentes dos processos matemáticos. [...] Nessas áreas, os conceitos e processos algébricos, como manipulação de variáveis e a avaliação de tendências, são de importância fundamental."

Assim, dificuldades no entendimento dos conceitos básicos da álgebra podem ocasionar futuros obstáculos mesmo que o educando não escolha um caminho profissional diretamente relacionado às ciências exatas. A álgebra, usada tanto na matemática pura quanto na aplicada, tem fundamental importância. "Uma das potencialidades da Álgebra advém da utilização de símbolos, muitos deles literais. Estes permitem expressar idéias matemáticas de forma rigorosa e condensada e são ferramentas importantes para a resolução de problemas." (Matos, 2007, p. 12).

Neste artigo, o objetivo é refletir sobre o ensino dos conceitos iniciais da álgebra, pesquisar o entendimento de estudantes de Licenciatura em Matemática sobre os conceitos elementares da álgebra e apresentar possibilidades de superação das dificuldades

\section{Concepções de álgebra e de variável}

Para Usiskin (1995, p. 9), a álgebra da escola média se relaciona à "compreensão do significado das letras (hoje comumente chamadas de variáveis) e das operações com elas, e consideramos que os alunos estão estudando álgebra quando encontram variáveis pela primeira vez."

Silva (2009), ao pesquisar sobre os diferentes usos da variável, relata que as primeiras investigações sobre o uso da letra na álgebra escolar foram apresentadas por Küchemann, a partir da pesquisa CSMS (Concepts in Secondary Mathematics and Science), realizada na Inglaterra no período entre 1974 e 1979. Através da aplicação de um questionário e de entrevistas, foi possível identificar diferentes utilizações das letras por parte dos alunos. Küchemann caracterizou seis tipos de interpretações: letra calculada (ou avaliada), letra ignorada ou não usada, letra usada como objeto, letra como incógnita, letra generalizando números e letra usada como variável. Silva (2009) analisou esta pesquisa de Küchemann, descrevendo cada uma das interpretações e enfocando alguns exemplos conforme se apresenta a seguir.

$\mathrm{Na}$ categoria letra avaliada, as letras tem um valor específico, um valor numérico. São inicialmente desconhecidas, mas calculáveis por substituição através de contagem na qual não se opera diretamente com as letras como incógnitas. Alguns exemplos que se enquadram na categoria são: O que se pode dizer sobre $a$, se $a+5=8$ ? O que se pode dizer sobre $u$, se $u=v$ +3 e $v=1$ ? 
Quando as letras não são usadas ou ignoradas, não é associado qualquer significado. A focalização ocorre nos processos aritméticos como no exemplo Se $a+b=43$, então $a+b+$ $2=\ldots$ no qual a resolução ocorre pelo processo $43+2$.

Em letra usada como objeto, as letras são vistas como objetos concretos ou mesmo como abreviações dos objetos, trocando-se o seu significado abstrato por algo concreto. Um exemplo utilizado é o de perímetro, em que a letra é entendida como sendo o lado do polígono. Essa identificação de letra usada como objeto da realidade torna a simplificação de expressões algébricas algo intuitivo para o estudante, pois em situações concretas apenas adiciona-se objetos de mesma natureza. No entanto, esta visão pode trazer dificuldades quando a situação é desprendida da realidade.

$\mathrm{Na}$ categoria letra usada como incógnita, ela é considerada como número desconhecido específico, com o qual se pode operar diretamente. A letra é usada como incógnita no exemplo $O$ que se pode dizer sobre $r$, se $r=s+t$ e $r+s+t=30$ ? Neste exemplo, opera-se de maneira a substituir $s+t$ por $r$ na segunda expressão.

Em letras generalizando números, elas são percebidas mais como uma representação de vários valores numéricos do que exatamente um valor. No exemplo, $O$ que podemos dizer sobre $c$, se $c+d=10$ e cé menor do que $d$ ?, os alunos apresentaram respostas como $c<5$; $c$ $=1,2,3,4$ ou $c=10-d$.

Pesquisas a respeito dos usos da variável foram desenvolvidas no México por Ursini e Trigueros. Essas autoras, juntamente com Reyes e Quintero, apresentam três maneiras pelas quais a variável normalmente é utilizada na álgebra escolar e as caracterizam da seguinte maneira:

\footnotetext{
[...] a variável como incógnita, cujo valor pode ser determinado com precisão tendo em consideração as restrições do problema; a variável como número geral, ou seja, o que aparece em generalizações e métodos em geral; e variáveis em uma relação de variação conjunta com outras variáveis que denominamos variável em relação funcional. (TRIGUEROS et al, 1996, p. 352, tradução nossa)
}

As habilidades envolvidas no tratamento da variável, segundo o modelo denominado por Trigueros, Quintero, Reyes, Ursini (1996, p. 317), os três usos da variável, são apresentadas caracterizando a simbolização, a interpretação e a manipulação. $\mathrm{Na}$ variável como incógnita, a simbolização ocorre para um termo desconhecido em uma situação particular e/ou em uma equação onde a incógnita aparece uma ou mais vezes e faz-se necessário manipular com fatoração, simplificações e balanceamento de equações para calcular o valor numérico da variável da equação. A simbolização de um objeto geral, envolvido em métodos ou regras gerais, deduzidos de padrões numéricos e/ou geométricos, ou em famílias de problemas similares, ocorre quando a variável é tratada como número geral presente em expressões algébricas onde a fatoração, simplificação e a reorganização estão presentes. A variável em relações funcionais ocorre quando da interpretação da correspondência entre variáveis em expressões algébricas, tabelas e gráficos ou em problemas em língua usual. Nestas situações a fatoração e simplificação são utilizadas para reorganizar uma expressão, sendo necessário, em alguns casos, substituir valores para determinar intervalos de variação, valores de máximo e mínimo ou analisar o comportamento global da relação.

Investigando as concepções de variável, Usiskin apresenta o entendimento de diversos autores de textos matemáticos. O autor identifica que, em livros publicados na década de 1950, ocorre a concepção de variável como número mutável, letra que representa um número e símbolo que é substituído pelo nome de alguns objetos, normalmente números em álgebra. 
Considera, ainda, que a concepção de variável muda com o tempo, sendo que, na atualidade, a concepção de variável como um símbolo que representa indistintamente os elementos de um conjunto é raramente questionada. Conclui, por fim, que "as finalidades da álgebra são determinadas por, ou relacionam-se com, concepções diferentes da álgebra que correspondem à diferente importância dada aos diversos usos das variáveis." (Usiskin, 1995, p.13).

Apresenta-se a seguir as quatro concepções de álgebra identificadas por Usiskin (1995): aritmética generalizada, um estudo de procedimentos para resolver certos tipos de problemas, estudo de relações entre grandezas e estudo de estruturas.

Se a álgebra é concebida como aritmética generalizada, então as variáveis são generalizadoras de modelos. Por exemplo, dados numéricos coletados em uma pesquisa e relacionados podem ser descritos matematicamente. Nessa concepção de álgebra, as variáveis são empregadas para traduzir e generalizar situações que envolvem relações entre grandezas.

\begin{abstract}
Num nível mais avançado, a noção de variável como generalizadora de modelos é fundamental em modelagem matemática. Muitas vezes encontramos relações entre números que desejamos descrever matematicamente, e as variáveis são instrumentos utilíssimos nessa descrição. [...] Dentro dessa concepção de álgebra, as instruçõeschave para o aluno são traduzir e generalizar. (Usiskin, 1995, p.13)
\end{abstract}

A álgebra como generalizadora de modelos é de fundamental importância para generalizar situações da aritmética, pois descrições de procedimentos aritméticos em linguagem usual não são de fácil compreensão. Por exemplo, a regra da multiplicação de frações, pode ser descrita na linguagem usual como sendo o resultado da multiplicação de duas frações é obtido da seguinte forma: o numerador é resultado da multiplicação dos numeradores das duas frações e o denominador é resultado da multiplicação dos denominadores das duas frações. Tal frase é de difícil entendimento, enquanto que em linguagem algébrica a descrita da regra torna-se clara: $\frac{a}{c} \times \frac{b}{d}=\frac{a \times b}{c \times d}$.

Se a álgebra é entendida como um estudo de procedimentos para resolver certos tipos de problemas, então as variáveis são ou incógnitas ou constantes. Inicialmente, os problemas propostos são traduzidos para a linguagem da álgebra, sendo utilizados procedimentos para simplificar e resolver. Por exemplo, é solicitado que se adicione 3 ao quíntuplo de um determinado número para que a soma seja 40. Para que esse número seja calculado, o problema é traduzido em linguagem algébrica, $5 x+3=40$, e são efetuados procedimentos para resolver a equação. Nesse caso, a letra $x$ representa um valor desconhecido que pode ser determinado empiricamente ou por meio de alguma técnica, o que caracteriza a variável enquanto incógnita. A tradução da situação em linguagem algébrica é apenas o primeiro passo, pois, seguidamente, deve-se tratar da equação obtida determinando o valor ou os valores possíveis para a incógnita.

Na concepção de álgebra como estudo de relações entre grandezas, a variável não é um valor que será calculado. Exemplos: (a) três grandezas estão relacionadas na fórmula da área de um retângulo, $\mathrm{A}=\mathrm{bh}$; (b) o que ocorre com o valor de $\frac{1}{\mathrm{x}}$ quando $x$ assume valores cada vez maiores. Nesses exemplos não se está buscando o valor numérico de $x$. Nessa concepção de álgebra, a variável é um argumento representando os valores do domínio de uma função ou um parâmetro, um número do qual dependem outros números.

Nos cursos superiores, a álgebra é estudo de estruturas e a variável é um símbolo arbitrário. Por exemplo, se uma identidade trigonométrica está sendo mostrada, não se 
entende a variável como um número a ser calculado, nem estão sendo consideradas as funções envolvidas, mas utilizam-se propriedades e as variáveis são manipuladas.

Assim, diante do diálogo com Silva, Ursini, Trigueros e Usiskin, constata-se a importância do entendimento das diversas concepções de variável em álgebra, dependendo do contexto em que está inserida.

\section{Dificuldades no estudo da álgebra}

As pesquisas de Booth (1995) identificam as principais áreas em que os estudantes manifestam dificuldades em álgebra: a interpretação de letras, a formalização dos métodos utilizados e sua relação com os métodos da aritmética e a compreensão de notação e de convenções.

O foco da atividade algébrica é estabelecer relações entre grandezas e expressá-las de forma simplificada, de forma geral. Apesar de efetuarem-se alguns procedimentos para resolver problemas, que por vezes resultam em uma resposta numérica, o foco principal e imediato da álgebra é o estabelecimento da generalização. Já o foco da atividade aritmética é o de obter respostas numéricas particulares, o que faz com que muitos alunos procurem um resultado numérico para as expressões algébricas. Muitos discentes, ao enfrentarem problemas algébricos, conseguem chegar a uma expressão algébrica correta, porém não a consideram uma resposta adequada. Reagem com estranheza e perguntam ao professor se realmente acertaram, pois não se convencem de estarem diante da resposta ao problema. Outros, por sua vez, inclinam-se em juntar todos os termos da expressão algébrica obtida de qualquer maneira, pretendendo como resposta um único termo, assim como em aritmética tem-se como resposta um único número. Essas constatações são oriundas de nossa vivência pedagógica e são abordadas nas pesquisas de Booth (1995).

A dificuldade dos estudantes não aceitarem uma resposta algébrica aberta, ou seja, que a resposta seja apresentada na forma de um polinômio, é denominada por Booth (1995) como dificuldade em aceitar a ausência de fechamento e ocorre na passagem da aritmética para álgebra, ocasionando erros na compreensão de situações algébricas. Por exemplo, expressões do tipo $2 \mathrm{a}+4 \mathrm{~b}$ são comumente entendidas pelos estudantes como sendo equivalente a $6 \mathrm{ab}$ e esse erro pode ter origem na dificuldade em aceitar a ausência de fechamento. Essa dificuldade em aceitar respostas algébricas se deve à interpretação equivocada das operações aritméticas. No exemplo acima, os estudantes procuram adicionar algebricamente termos não semelhantes, pois em aritmética é comum a resposta de um único termo, onde o sinal de adição é unidirecional, entendido como desenvolvimento de uma ação.

A mudança no significado de justaposição e a ampliação do significado dos sinais operatórios, na passagem da aritmética para álgebra, são fontes de concepções erradas em álgebra. A justaposição em aritmética é utilizada para a formação de números e é entendida como a adição dos valores dos algarismos que compõem o número, que dependem da posição que ocupam. Para exemplificar, o número 23 é entendido como a adição de $20+3$. Em álgebra, a justaposição é entendida como multiplicação entre letras ou mesmo entre números e letras. Por exemplo, 2a significa a multiplicação de 2 por a. Devido à compreensão de justaposição em álgebra diferir da interpretação enraizada por anos de estudo da aritmética, é recomendável registrar a operação de multiplicação entre letras e números como ocorre na aritmética, simbolizada por $(\times)$ ou $(\cdot)$, para gradualmente inserir a justaposição no sentido algébrico. Apresentam-se a seguir considerações de Booth $(1995, \mathrm{p} .29)$ referindo-se à maneira de representar a multiplicação em álgebra por justaposição: 
A tendência aparentemente forte de as crianças verem isso como soma em vez de produto (ou como representação de valor posicional) pareceria indicar que sua introdução deveria ser retardada e que o produto deveria ser escrito na forma completa (n $\times 3$ ou $3 \times n$ ) por um período considerável da fase de iniciação dos alunos em álgebra.

Parte da dificuldade em simplificar expressões algébricas está na relação entre o significado dos símbolos operatórios em álgebra e aritmética, pois os sinais operatórios são interpretados, geralmente, como ações que devem ser efetuadas. O símbolo de adição é comumente entendido como realizar a operação, juntar os números. Em álgebra, no entanto, o sinal de adição indica uma ação, quando os termos possuem a mesma parte literal, ou implica em representar resultado de uma adição quando os termos possuem a parte literal diferente. Por exemplo, no caso da expressão $3 x+2 x$ o sinal indica uma ação e espera-se que o estudante junte os termos semelhantes e obtenha $5 x$, já em $4 \mathrm{a}+2 \mathrm{~b}$, o sinal indica que a expressão algébrica é resultado de uma adição.

Também ocorrem equívocos no entendimento do símbolo de igualdade, não compreendido como sinal de equivalência, mas como indicativo de que seja escrita uma resposta, o que comumente ocorre em aritmética. Em álgebra, é imprescindível que o sinal de igualdade seja entendido no seu valor bidirecional, para que os procedimentos utilizados para resolução de equações não se tornem mecânicos e sem explicação. Por isso, é importante que desde as primeiras experiências dos estudantes os símbolos operatórios sejam lidos de forma adequada. O sinal de igualdade exige uma leitura como é igual a ao invés de dá, como geralmente é lido. Por exemplo, a igualdade $2+3=5$ é lida geralmente como $2+3$ dá 5 e é importante que, para explicitar o sentido bidirecional de equivalência entre os membros da igualdade, seja lida como $2+3$ é igual a 5 .

É necessário que o entendimento do sinal de adição não seja apenas como uma instrução, mas também como resultado. Para isso, a leitura de, por exemplo, $2+3$, que é lido, normalmente, apenas como 2 mais 3 ou some 2 com 3, pode ser interpretado como "o número que é 3 mais que 2", conforme indica Booth.

Essas concepções têm origem no que Booth (1995) denomina dilema nome-processo.

\footnotetext{
Por exemplo " $n+3$ " pode ser uma expressão de uma "instrução" (ou procedimento), que afirma que se deve somar 3 à variável n, e também uma "resposta", que dá o resultado de se ter efetuado uma adição. No primeiro caso pode-se interpretar a expressão como "some 3 a n"; no segundo como "o número que excede n em 3 unidades". (BOOTH, 1995, p.27)
}

Booth sugere que essas dificuldades podem ser contornadas quando tratadas com expressões algébricas em atividades concretas. Por exemplo, é aceita a expressão $2 \mathrm{a}+4 \mathrm{~b}$ quando apresentada da forma perímetro $=2 a+4 b$. Este autor constatou que a simbologia algébrica deve ser introduzida de forma cautelosa, sempre fazendo um paralelo à aritmética e à escrita em linguagem usual do significado da expressão.

Booth (1995) aponta outra dificuldade bem comum, que consiste na leitura incorreta de letras em álgebra, entendidas como representando um objeto ao invés de identificar como sendo uma variável, não expressando adequadamente seu significado. Por exemplo, expressões do tipo 6a, onde $a$ representa a quantidade de acerolas, é comumente lida como 6 acerolas. Nessa leitura, $a$, que representa uma variável na expressão, não tem esse significado pois, na leitura de 6 acerolas, a quantidade está definida, não há a ideia de variação. 
A leitura inadequada pode induzir a procedimentos incorretos ao lidar com expressões algébricas. Muitos estudantes, por exemplo, justificam na leitura de $6 a$ como 6 acerolas e $5 m$ como 5 maçãs, a simplificação de $6 a+5 m$ como 11 am e leem o resultado como 11 acerolase-maçãs. Se a leitura fosse realizada de forma adequada, deixando-se explícita a ideia de variação na quantidade de cada fruta, a simplificação conflitaria com a descrição de cada termo separadamente.

Focando a prática pedagógica do professor de matemática frente a situações de erro ou dificuldades em situações de aula envolvendo álgebra elementar, Pinto e Fiorentini (1997) realizaram um estudo de caso com uma turma da $7^{\mathrm{a}}$ série do ensino fundamental. Os autores focaram o processo de transcrição a partir de uma situação problema da linguagem usual para a linguagem algébrica. Por meio de entrevista, a professora explicita a importância de entender o significado das letras para depois começar realmente a álgebra. Tal depoimento é analisado pelos autores concluindo-se que a professora parece não entender a fase inicial da construção de significados da linguagem algébrica como parte constituinte da própria álgebra, que é entendida como cálculo literal. Foi proposto para os alunos escreverem em linguagem algébrica $O$ quadrado da coisa mais um é igual a 10. Qual o valor da coisa? Quando a professora perguntou se lembravam de questões sobre valor desconhecido, os alunos foram unânimes em afirmar que lembravam do quadradinho desenhado nas séries iniciais como símbolo para representar este valor. O significado da palavra quadrado explicitado pelos alunos se relacionava às atividades das séries iniciais, de modo que, ao efetuarem a transcrição solicitada, apresentaram $\square+1=10$. Não observando os registros escritos pelos estudantes, a professora resolve a questão no quadro-negro, explicando que vai engaiolar a coisa: $(\text { coisa })^{2}+1=10$, provocando reações maliciosas nos alunos. Não foi apresentado o significado histórico da palavra coisa, a qual foi utilizada por matemáticos na época em que ainda não usavam linguagem simbólica. Neste caso, Pinto e Fiorentini (1997, p.67) consideram que "a professora, tentando inovar, utiliza um recurso didático e dá a impressão que é para tentar aproximar-se/facilitar a compreensão do aluno". E nesta contextualização da professora há um desvio tanto do significado historicamente produzido quanto daquele veiculado no contexto matemático.

No estudo de caso acima apresentado, não foi priorizado o entendimento dos estudantes sobre o conceito do valor desconhecido, não ocorrendo um dialogo inicial com os educandos para conhecer suas concepções. A esse respeito discutem Lochhead e Mestre (1995, p.153):

\footnotetext{
Parece-nos que seria proveitoso os professores integrarem em suas técnicas de ensino uma tentativa de diagnosticar e tratar concepções erradas. Só então estaríamos certos de que os alunos caminhariam para a matemática de nível superior com o mínimo possível de bagagem adversa.
}

Antes de trabalhar com os educandos formas simbólicas, Schoen destaca a importância de descrever, em linguagem usual, atividades que posteriormente são formalizadas em linguagem algébrica. Seguindo a recomendação de Schoen (1995), é importante privilegiar a utilização da língua usual, conduzindo os educandos, gradualmente, da verbalização ao simbolismo algébrico:

A maioria dos alunos é capaz de entender a língua falada e escrita quando começa o primeiro ano de álgebra. Deveríamos tirar proveito desta capacidade escrevendo e falando em nossa língua sobre idéias matemáticas, antes da introdução do simbolismo e paralelamente a ela. (Schoen, 1995, p.138)

Isso requer que o professor considere os métodos informais usados pelos estudantes na busca de soluções, apresentando posteriormente situações nas quais eles se deparem com a limitação desses métodos. Assim, a álgebra passaria a ser utilizada por eles como facilitadora na resolução do processo. 


\section{Metodologia e análise da pesquisa}

Visando identificar as dificuldades que universitários encontram na compreensão da linguagem algébrica, apontadas nas pesquisas de Booth (1995), Lochhead e Mestre (1995), foram elaboradas cincos questões para serem respondidas por estudantes universitários. Para avaliar sua formação básica na linguagem e nos procedimentos algébricos, e ainda verificar se há dificuldades no entendimento dos conceitos iniciais da álgebra semelhantes aos relatados pelos referidos autores. Para tanto, as questões foram redigidas sempre apresentando diretamente o comando de traduzir para uma equação a situação dada, como realizado na maioria dos livros didáticos utilizados na educação básica que fizeram parte da formação da maioria dos pesquisados.

O questionário foi aplicado inicialmente em 2009 com nove acadêmicos da primeira fase do curso de licenciatura em Matemática da Universidade Regional de Blumenau. No ano de 2013, durante um minicurso realizado na Semana da Matemática, outros 36 estudantes de diversas fases do curso de Matemática responderam as mesmas questões. A seguir descrevemse as questões que foram aplicadas no teste com um relato das principais dificuldades observadas.

QUESTÃO 1 - Foi solicitada a descrição de uma situação através de linguagem algébrica, onde os dados necessários para a resolução são apresentados em uma figura.

De um avião, um homem tira uma fotografia de algumas vacas e porcos que estão num campo. Ele tem certeza que fotografou uma amostra típica dos animais desse campo. Escreva uma equação com as letras $V$ e $P$ para descrever a relação entre a quantidade $V$ de vacas e a quantidade $P$ de porcos no campo. Essa equação lhe permitirá calcular o número de vacas, dado o número de porcos.

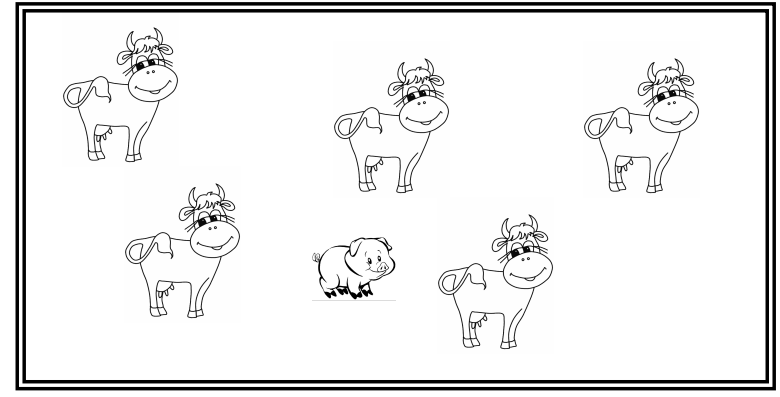

No primeiro grupo (em 2009) apenas $45 \%$ e no segundo grupo (2013) apenas $42 \%$ dos participantes escreveram a resposta adequada para a situação, ou seja, V $=5 \mathrm{P}$. Em geral, muitos pensam "para cada cinco vacas temos um porco" e descrevem algebricamente a situação seguindo a quantidade de animais visualizados, respondendo $\mathrm{P}=5 \mathrm{~V}$, fícando evidente o uso das letras para simbolizar objetos, $5 \mathrm{~V}$ cinco vacas e $\mathrm{P}$ para porcos.

No enunciado do problema, explicitou-se que a resposta é descrita por uma equação, porém alguns participantes não utilizaram uma descrição que contenha igualdade, ou seja, ou ignoraram a instrução do enunciado ou desconhecem o significado de equação, conforme pode-se observar a seguir.

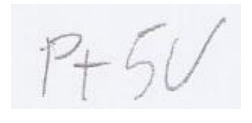


As respostas incorretas não são decorrentes da interpretação inadequada do enunciado, pois a equação escrita depende apenas de relação das quantidades observadas na figura. Parece coerente aos participantes $5 \mathrm{~V}$ como parte integrante de sua resposta, pois a leitura incorreta como cinco vacas, sugere exatamente o que se vê na figura.

QUESTÃO 2 - Com essa questão, pretendia-se avaliar exclusivamente a transcrição decorrente da leitura de uma situação em linguagem usual, conforme segue:

Escreva uma equação, usando as variáveis $C$ e $A$, que indicam, respectivamente, a quantidade de crianças e a quantidade de adultos, para representar a seguinte afirmação: "Há seis vezes mais crianças do que adultos neste parque".

A resposta adequada, $\mathrm{C}=6 \mathrm{~A}$, foi escrita apenas $22 \%$ dos participantes do primeiro grupo e por $39 \%$ do segundo grupo e a resposta mais frequente foi $\mathrm{A}=6 \mathrm{C}$, onde evidencia-se que a ordem das palavras no texto foi utilizada para descrever a ordem das variáveis na equação.

A escrita em linguagem usual é aquela que os estudantes usam com maior frequência e que influencia a leitura de qualquer outra linguagem, inclusive a algébrica. É possível observar que a escrita da linguagem usual ocorre da esquerda para a direita, o que é exatamente a ordem utilizada, por alguns participantes, para transcrever a situação.

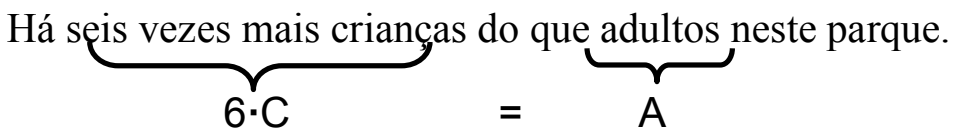

A tradução da situação da linguagem usual para a algébrica não ocorre de forma linear, pois é necessário avaliar numericamente ou quantitativamente a relação entre as grandezas quantidade de crianças e quantidade de adultos, ao invés de se realizar uma leitura linear da sequência de palavras. (Poffo, 2011, p. 128)

A resposta abaixo é destacada, pois verifica-se que a multiplicação é utilizada para relacionar as quantidades variáveis, o que demonstra a tendência de encontrar um fechamento para expressão, assim como em aritmética onde a solução é sempre apresentada em um único termo.

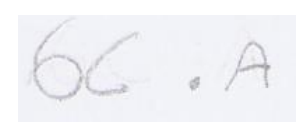

Nesta questão ficam evidentes as dificuldades de transcrição da linguagem usual para a algébrica, leitura incorreta das variáveis e a dificuldade de aceitar ausência de fechamento, conforme relatado nas pesquisas de Booth (1995).

QUESTÃO 3 - Nesta questão verifica uma relação de proporção para as quantidades variáveis envolvidas.

Escreva uma equação usando as variáveis T e C para indicar a quantidade de tortas e a quantidade de cucas, respectivamente, para representar a seguinte afirmação: "Na padaria da Joana, para cada quatro pessoas que pedem torta, cinco pedem cuca". foi:

Resposta escrita por $98 \%$ dos participantes do primeiro grupo e $92 \%$ do segundo grupo 


$$
4 t=5 c
$$

A resposta correta seria obtida evidenciando a proporção entre as quantidades:

$$
\frac{\mathrm{T}}{\mathrm{C}}=\frac{4}{5} \Leftrightarrow 5 \mathrm{~T}=4 \mathrm{C}
$$

A leitura das variáveis como objetos tortas e cucas ao invés de como quantidades variáveis e associação com a ordem das palavras no texto, ficam evidentes nesta questão. Claramente percebe-se que os estudantes não relacionam as linguagens aritmética e algébrica, pois um teste numérico na equação escrita apontaria o erro cometido, o que indica a presença da mecanização da álgebra e a imediata tradução, sem qualquer questionamento sobre o significado do resultado obtido.

Ressalta-se ainda, que um dos participantes escreveu uma equação, porém não como uma igualdade de proporções, mas sim na tentativa de dar um fechamento a expressão obtida.

Essa resposta verifica novamente a necessidade de que expressão resulte em um único termo - o resultado de uma igualdade, observando a dificuldade em compreender o significado da simbologia utilizada.

QUESTÃO 4 - A questão pretende verificar como são realizadas as leituras das variáveis, da justaposição e do sinal de adição em álgebra.

Escreva uma sentença em português que dê a mesma informação de cada expressão abaixo sabendo que a representa a quantidade de acerolas e $\boldsymbol{m}$ representa o número de maçãs:
(i) $2 a$
(ii) $3 m$
(iii) $2 a+3 m$
(iv) $5 \mathrm{am}$

As letras sendo interpretadas como representações de quantidades variáveis foram descritas adequadamente por $11 \%$ dos participantes do primeiro grupo e por $41 \%$ do segundo grupo, os demais fizeram a leitura das letras como representantes de objetos, não apresentando qualquer verificação da ideia de variável.

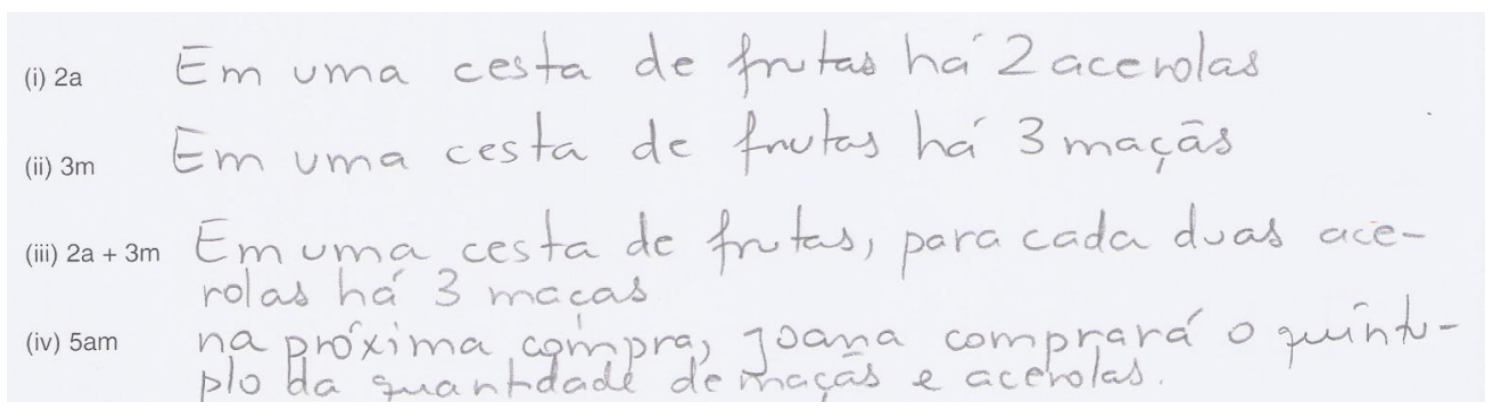

Booth (1995, p.31) observou, em sua pesquisa realizada no período de 1980 a 1983 no Reino Unido, a mesma leitura inadequada para as variáveis e indicou que "É preciso tomar cuidado quando se introduzem afirmações do tipo "a representa número de abacaxis", levando o aluno a converter " $3 \mathrm{a}$ " em "3 abacaxis", em vez de " 3 vezes o número de abacaxis." 
Booth (1995, p.29) explica que a leitura inadequada pode influenciar na simplificação incorreta de expressões algébricas fazendo parecer razoável que "[...] $2 a+5 b=7 a b$ (duas maçãs mais 5 bananas é igual a 7 maçãs-e-bananas)".

O quadro abaixo apresenta um panorama geral dos resultados obtidos, indicando a porcentagem de respostas inadequadas apresentadas:

\begin{tabular}{lcc}
\hline & Grupo 1 & Grupo 2 \\
\hline Questão 1 & $55 \%$ & $58 \%$ \\
Questão 2 & $78 \%$ & $61 \%$ \\
Questão 3 & $98 \%$ & $92 \%$ \\
Questão 4 & $89 \%$ & $58 \%$ \\
\hline
\end{tabular}

Os erros acima descritos também foram relatados em pesquisas realizadas por Lochhead e Mestre (1995) nos Estados Unidos. Estes pesquisadores comentam que resultados semelhantes foram obtidos com alunos hispânicos, da ilha de Fiji, de Israel e do Japão na década de 1980:

\begin{abstract}
Parece, portanto, que o ensino nos Estados Unidos, em Israel e em Fiji - e, acreditamos, em quase toda parte - não oferece aos alunos oportunidades de aprender a interpretar seqüências de símbolos matemáticos. Os alunos não aprendem a ler e a escrever em matemática! Essa omissão não só limita seu desempenho a resolução de problemas, como também os coloca em séria desvantagem quando se trata de aprender a manipulação simbólica das regras da álgebra. Sem a capacidade de interpretar expressões, os alunos não dispõem de mecanismos para verificar se um dado procedimento é correto. Assim, muitas vezes eles têm de recorrer a lembranças dos procedimentos automatizados para resolver problemas. (LOCHHEAD; MESTRE, 1995, p.148)
\end{abstract}

Dos resultados pode-se concluir que a maioria dos participantes dessa pesquisa utilizam a álgebra como um procedimento mecanizado, pois não demonstram analisar se suas expressões tem coerência com o problema apresentado, o que poderia ser realizado com verificação numérica.

As atividades propostas para contribuir no processo de compreensão da linguagem algébrica foram desenvolvidas com base na metodologia proposta por Lochhead e Mestre (1995) onde utiliza-se uma abordagem qualitativa, quantitativa e por fim conceitual, fazendo com que o estudante seja conduzido ao pensamento algébrico a partir da aritmética e que concepções conceitualmente inadequadas sejam verificadas e superadas pelo próprio estudante.

Normalmente quando se fala em álgebra os estudantes imediatamente associam a operação com letras como significado para a palavra, porém como sugere a própria construção histórica, a descrição com palavras de uma variação também implica em linguagem algébrica. Conforme Boyer (1996), o desenvolvimento da notação algébrica evoluiu ao longo de três estágios: o primitivo ou retórico, completamente verbal e escrito com palavras; o intermediário ou sincopado em que são adotadas algumas abreviações; e o final ou simbólico, em que as expressões são sintetizadas por símbolos.

Nas escolas de educação básica, esta mesma simbolização em álgebra, que levou mais de 3000 anos para acontecer, normalmente ocorre rapidamente, descartando o processo de construção histórico da mesma. Segundo Schoen (1995, p.138) "lançar os educandos precipitadamente ao simbolismo algébrico é ignorar a necessidade de uma fundamentação 
verbal e de uma simbolização gradual sugeridas pela construção histórica da álgebra." Assim também, a ruptura do pensamento aritmético - que fortemente predomina durante 6 a 7 anos antes de estudantes aprenderem álgebra pela primeira vez - para o algébrico, se não for concebido com cautela, pode contribuir para potencializar as dificuldades observadas no estudo da álgebra.

\section{Possibilidades de superação das dificuldades encontradas pelos estudantes}

Após a realização da pesquisa acima relata ofereceu-se um curso de aperfeiçoamento de 40 horas que ocorreu em regime semi-presencial, durante o período de 27 de setembro a 11 de outubro de 2013, onde foram propostas atividades que seguem a metodologia de Lochhead e Mestre (1995). Este curso foi realizado dividindo-se as atividades nos seguintes módulos:
a) compreensão de variável;
b) simbolização de variável;
c) compreensão de incógnita;
d) potenciação em álgebra;
e) atividades algébricas resolvidas aritmeticamente;
f) raciocínio de generalização com o uso de computador.

O foco principal da resolução de todas as questões propostas no curso foi a realização de questionamentos, iniciando por uma abordagem de ordem qualitativa do tipo:

a) Quais as grandezas envolvidas no problema?

b) Quais grandezas mudam e quais não mudam?

Aos poucos, alterando a última pergunta para "Quais grandezas são constantes e quais são variáveis?".

Em seguida questionamentos quantitativos para as variáveis do problema como "se a grandeza tal fosse de tantas unidades quanto teríamos da outra grandeza?", ou ainda, "alguma grandeza estará sempre em maior quantidade que outra?".

Os questionamentos conceituais não necessitam de simbolização. Sugere-se para as primeiras questões a solicitação que, usando suas palavras, o estudante descreva como se pode obter a quantidade de uma grandeza em decorrência da outra para posteriormente introduzir a simbolização. Por fim, sugere-se induzir para retomada e verificação da expressão obtida indicando que sejam substituídos valores numéricos utilizados na abordagem quantitativa e verificado a coerência do resultado obtido.

É importante que as questões algébricas presentes nos livros didáticos sejam resolvidas cautelosamente priorizando a utilização da linguagem usual evitando a algebrização precoce

\section{Considerações finais}

A pesquisa realizada revelou que estudantes de cursos universitários encontram dificuldades no entendimento das diversas concepções de álgebra que estão relacionadas com os diferentes entendimentos de variável. A análise dos dados apontou leitura incorreta das variáveis, equívocos no uso da notação algébrica e na compreensão das relações da álgebra com a aritmética e com a língua usual. 
Constata-se ser importante, no estudo inicial da álgebra, dedicar especial atenção ao conceito de variável através de situações cotidianas que envolvam a ideia de mudança, e, progressivamente, realizar a transcrição de situações descritas em linguagem usual para a linguagem algébrica. Tradicionalmente, o símbolo da multiplicação não é explicitado, cabendo ao estudante deduzir que a ausência de operador significa uma operação de multiplicação. No entanto, é prudente escrever o símbolo da multiplicação, pois muitos estudantes confundem a justaposição de algarismos e letras simbolizando variáveis com a operação adição da aritmética.

$\mathrm{Na}$ transcrição de situações descritas na linguagem usual para a linguagem algébrica, muitos estudantes realizam uma equivocada associação entre a ordem das palavras e a escrita da equação correspondente. A metodologia de questionamento sugerida por Lochhead e Mestre (1995) mostrou ser adequada para a superação das dificuldades na transcrição de situações pois permite o entendimento do problema ser resolvido de forma qualitativa e quantitativa.

Espera-se que este artigo contribua para uma reavaliação da prática pedagógica, possibilitando uma reflexão sobre modos de nortear o estudo dos conceitos iniciais da álgebra, para que as dificuldades encontradas pelos estudantes sejam minimizadas.

\section{Referências}

Booth, L. R. (1995). Dificuldades das crianças que iniciam álgebra. In: Coxford, Arthur F.; Shulte, A. P. (Org.). As Idéias da Álgebra. São Paulo: Atual, p.23-26.

Boyer, C. B. (1996). História da Matemática. 2 ed. São Paulo: Edgard Blucher.

House, P. A. (1995). Reformular a álgebra da escola média: por que e como?. In: Coxford, Arthur F.; Shulte, A. P. (Org.). As Idéias da Álgebra. São Paulo: Atual, p.1-8.

Lochhead, J.; Mestre, J. P. (1995). Das palavras à álgebra: corrigindo concepções erradas. In: Coxford, A. F.; Shulte, A. P. (Org.). As Idéias da Álgebra. São Paulo: Atual, p.144-154.

Matos, A. S. S. M. (2007). Explorando relações funcionais no $8^{\circ}$ ano: um estudo sobre o desenvolvimento do pensamento algébrico. 256 f. Dissertação (Mestrado em Educação) - Universidade de Lisboa, Portugal.

Pinto, R. A. \& Fiorentini, D. (1997). Cenas de uma aula de álgebra: produzindo e negociando significados para a "coisa". Zetetiké (Vol. 5, n.o 8, pp. 45-71). Campinas: UNICAMP FE/CEMPEM.

Poffo, Janaína. (2011). Álgebra nos Anos Finais do Ensino Fundamental: Reflexões e Atividades Pedagógicas. 143f. Dissertação (Mestrado em Ensino de Ciências Naturais e Matemática) - Universidade Regional de Blumenau.

Schoen, H. L. (1995). Ensinar a álgebra elementar focalizando problemas. In: Coxford, A. F.; Shulte, A. P. (Org.). As Idéias da Álgebra. São Paulo: Atual, p.135-144.

Silva, M. da. (2009). Diferentes usos da variável por alunos do Ensino Fundamental. 2009. Dissertação (Mestrado em Educação Matemática) - Pontifícia Universidade Católica de São Paulo, São Paulo. 
Trigueros M., Quintero R., Reyes A. \& Ursini S. (1996). Diseño de un cuestionario de diagnóstico acerca del manejo del concepto de variable em el álgebra. Enseñanza de las ciencias: revista de investigación y experiencias didácticas, Barcelona, Espanha, v.14, n.3, p.351-364.

Usiskin, Z. (1995). Concepções sobre a álgebra da escola média e utilizações das variáveis. In: Coxford, A. F.; Shulte, A. P. (Org.). As Idéias da Álgebra. São Paulo: Atual, p.9-22. 